\title{
Exploring Metallic Ratios
}

\author{
R. Sivaraman \\ Department of Mathematics, Dwaraka Doss Goverdhan Doss Vaishnav College, Chennai - 600 106, Tamil Nadu, India
}

Received April 20, 2020; Revised May 9, 2020; Accepted June 23, 2020

Copyright $\mathrm{C} 2020$ by authors, all rights reserved. Authors agree that this article remains permanently open access under the terms of the Creative Commons Attribution License 4.0 International License

\begin{abstract}
Huge amount of literature has been written and published about Golden Ratio, but not many had heard about its generalized version called Metallic Ratios, which are introduced in this paper. The methods of deriving them were also discussed in detail. This will help to explore further in the search of universe of real numbers. In mathematics, sequences play a vital role in understanding of the complexities of any given problem which consist of some patterns. For example, the population growth, radioactive decay of a substance, lifetime of an object all follow a sequence called "Geometric Progression". In fact, the rate at which the recent novel corona virus (COVID 19) is said to follow a Geometric Progression with common ratio approximately between 2 and 3 . Almost all branches of science use sequences, for instance, genetic engineers use DNA sequence, Electrical Engineers use Morse-Thue Sequence and this list goes on and on. Among the vast number of sequences used for scientific investigations, one of the most famous and familiar is the Fibonacci Sequence named after the Italian mathematician Leonard Fibonacci through his book "Liber Abaci" published in 1202. In this paper, I shall try to introduce sequences resembling the Fibonacci sequence and try to generalize it to identify general class of numbers called "Metallic Ratios".
\end{abstract}

Keywords Golden Ratio, Silver Ratio, Bronze Ratio, Metallic Ratios, Shift Operator, Recurrence Relation, Platinum Ratio, Rhodium Ratio

\section{Fibonacci Sequence and Golden Ratio}

Huge amount of work has been devoted to exploring the properties of Fibonacci sequence and Golden Ratio. So, I restrict myself to the essential ideas which were useful in proceeding for this paper. We first consider an interesting problem posed by Fibonacci in "Liber Abaci" ("The Book of Calculations")

Starting with one pair of rabbit, assuming that each pair produces a new pair after one month, assuming that none of the rabbit pairs die, how many rabbit pairs would be there at the end of one year?

The answer to this question lies in the sequence $1,1,2,3$, $5,8,13,21,34,55,89,144,233,377, \ldots$ Thus there would be 144 pairs of rabbits at the end of one year, as described by the sequence. This sequence is called "Fibonacci Sequence" named after the proposer though it is known to earlier mathematicians. This sequence has generated so much interest in mathematics especially to layman in conveying the beauty of mathematics. In fact, a separate journal named "Fibonacci Quarterly" is dedicated to study the properties of these fascinating numbers.

Considering $x_{n}$ to be the number of rabbit pairs generated in $n^{\text {th }}$ month, the terms of the sequence described above can be written as

$$
\begin{aligned}
& x_{1}=1, x_{2}=1, x_{3}=2, x_{4}=3, x_{5}=5, x_{6}=8, x_{7}=13, \\
& x_{8}=21, x_{9}=34, x_{10}=55, x_{11}=89, x_{12}=144, \cdots
\end{aligned}
$$

We find that, but for the first two terms, beginning from $x_{3}$, each term is sum of two preceding terms, leading us to form the recurrence relation $x_{n+2}=x_{n+1}+x_{n}, n \geq 1 \rightarrow(1)$, where $x_{1}=1, x_{2}=1$

We can solve (1) using the shift operator $\mathrm{E}$ defined by $\mathrm{E}^{r}\left(x_{k}\right)=x_{k+r}, k \geq 1, r \geq 1$.

Thus (1) can be written as $\left(\mathrm{E}^{2}-\mathrm{E}-1\right) x_{n}=0$. The auxiliary equation is: $m^{2}-m-1=0$. Solving this, we get two real and unequal roots $m=\frac{1 \pm \sqrt{5}}{2}$. Among these two roots, the number $\frac{1+\sqrt{5}}{2}$ is called the "Golden Ratio". If we denote this number by $\phi$ then the two roots of the auxiliary equation can be expressed as $1-\phi, \phi$.

Hence the solution to (1) can be expressed as $x_{n}=\alpha(1-\phi)^{n}+\beta \phi^{n}$ for $n \geq 1$.

From $x_{n}$ derived above, we can prove that the limit of the ratio of successive Fibonacci Numbers tends to the 
Golden Ratio $\phi=\frac{1 \pm \sqrt{5}}{2}$.

First we note that $1-\phi=\frac{1-\sqrt{5}}{2}=-0.618 \ldots,-1<1-\phi<1$.

Hence, $(1-\phi)^{n} \rightarrow 0$ as $n \rightarrow \infty$.

If we now consider the ratio of successive terms of the Fibonacci sequence then we get

$$
\begin{aligned}
& \quad \frac{x_{n+1}}{x_{n}}=\frac{\alpha(1-\phi)^{n+1}+\beta \phi^{n+1}}{\alpha(1-\phi)^{n}+\beta \phi^{n}} \rightarrow \frac{\alpha(0)+\beta \phi^{n+1}}{\alpha(0)+\beta \phi^{n}}=\phi \\
& n \rightarrow \infty \text {, proving the claim. }
\end{aligned}
$$

\section{Generating New Ratios}

\subsection{Let us now consider the sequence $1,1,3,7,17,41$, $99,239, \ldots$}

We see that $3=2(1)+1,7=2(3)+1,17=2(7)+3,41=$ $2(17)+7, \ldots$ So, the terms of this sequence form an recurrence relation given by $x_{n+2}=2 x_{n+1}+x_{n}, n \geq 1 \rightarrow(2)$, where $x_{1}=1, x_{2}=1$.

Solving (2) as we did in (1), using shift operator $\mathrm{E}$ we get $\left(\mathrm{E}^{2}-2 \mathrm{E}-1\right) x_{n}=0$. The auxiliary equation is $m^{2}-2 m-1=0$. Solving this, we get two real and unequal roots given by $m=1 \pm \sqrt{2}$. Among these two roots, the number $1+\sqrt{2}$ is called the "Silver Ratio". If we denote this number by $\lambda$ then the other root will be $2-\lambda$. Hence the solution of (2) can be written as $x_{n}=\alpha(2-\lambda)^{n}+\beta \lambda^{n}, n \geq 1$. As observed in the case of Golden Ratio, we can show that the ratio of successive terms of the sequence described through (2) approaches Silver Ratio $\lambda=1+\sqrt{2}$.

First we note that $-1<2-\lambda<1$ and so $(2-\lambda)^{n} \rightarrow 0$ as $n \rightarrow \infty$.

Thus, $\frac{x_{n+1}}{x_{n}}=\frac{\alpha(2-\lambda)^{n+1}+\beta \lambda^{n+1}}{\alpha(2-\lambda)^{n}+\beta \lambda^{n}} \rightarrow \frac{\alpha(0)+\beta \lambda^{n+1}}{\alpha(0)+\beta \lambda^{n}}=\lambda$ as $n \rightarrow \infty$.

\subsection{We now consider the sequence $1,1,4,13,43,142$, $469,1549,5116, \ldots$}

We see that $4=3(1)+1,13=3(4)+1,43=3(13)+4$, $142=3(43)+13, \ldots$ So, the terms of this sequence form an recurrence relation given by $x_{n+2}=3 x_{n+1}+x_{n}, n \geq 1 \rightarrow$ (3) where $x_{1}=1, x_{2}=1$.

Using the shift operator, equation (3) can be written as $\left(\mathrm{E}^{2}-3 \mathrm{E}-1\right) x_{n}=0$. The auxiliary equation is $m^{2}-3 m-1=0$. Solving this we get two real and unequal roots given by $m=\frac{3 \pm \sqrt{13}}{2}$. Among these two roots, the number $\frac{3+\sqrt{13}}{2}$ is called the "Bronze Ratio".

If we denote this number by $\mu$ then the other root will be $3-\mu$. Hence the solution of (3) can be written as $x_{n}=\alpha(3-\mu)^{n}+\beta \mu^{n}, n \geq 1$. Here also, it is easy (as proved in the case of Golden and Silver Ratios) that the ratio of the consecutive terms of the sequence described through (3) tends to the Bronze Ratio $\mu=\frac{3+\sqrt{13}}{2}$.

We thus have three ratios namely Golden Ratio $\phi=\frac{1 \pm \sqrt{5}}{2}$, Silver Ratio $\lambda=1+\sqrt{2}$, Bronze Ratio $\mu=\frac{3+\sqrt{13}}{2}$ described through the equations (1), (2) and (3) respectively. We also see that the ratio of the successive terms of the corresponding sequences approaches the respective ratios.

\section{Generalized Ratio}

We now try to generalize the recurrence relations obtained above to produce more general ratio from which Golden, Silver and Bronze ratios follow as special cases.

The more general recurrence relation is defined as $x_{n+2}=k x_{n+1}+x_{n}, n \geq 1 \rightarrow(4)$ where $x_{1}=1, x_{2}=1$. That is, after the first two terms which are 1, each term is $k$ times the previous term and added to last but one term. Forming a general equation using shift operator we get $\left(\mathrm{E}^{2}-k \mathrm{E}-1\right) x_{n}=0$. The auxiliary equation is $m^{2}-k m-1=0$. Solving this we find we get two real and unequal roots $m=\frac{k \pm \sqrt{k^{2}+4}}{2}$. Among these two roots, we call the number $\rho_{k}=\frac{k+\sqrt{k^{2}+4}}{2}$ as "Metallic Ratio" of order $k$. The other root is clearly $k-\rho_{k}$.

\subsection{Special Cases}

(i) If $k=1$, then we get $\rho_{1}=\frac{1+\sqrt{5}}{2}=\phi$, the Golden Ratio.

(ii) If $k=2$, then we get $\rho_{2}=\frac{2+\sqrt{8}}{2}=1+\sqrt{2}=\lambda$, the Silver Ratio.

(iii) If $k=3$, then we get $\rho_{3}=\frac{3+\sqrt{13}}{2}=\mu$, the Bronze Ratio.

Thus the Metallic Ratio $\rho_{k}=\frac{k+\sqrt{k^{2}+4}}{2}$ of orders 1, 2 and 3 are precisely the Golden, Silver and Bronze Ratios. This is in analogue with winning positions of medals in Olympics and other global sports. 
We observe that as $k$ is very large, $\rho_{k}$ also becomes very large. But we obtain interesting situations, if we assume some special values of $k$.

If $k=\lfloor\sqrt{2}\rfloor$ or $k=\lfloor\sqrt{3}\rfloor$ then $k=1$ giving Golden Ratio. Similarly, if $k=\lfloor e\rfloor$ then $k=2$ giving Silver Ratio and if $k=\lfloor\pi\rfloor$ then $k=3$ giving Bronze Ratio. Here $\lfloor x\rfloor$ is the largest integer $\leq x$ also called Floor Function of $x$.

\section{Behavior of Metallic Ratio}

Instead of considering natural number values for $k$, let us consider the case when $k$ is small given by $k=\frac{1}{n^{p}}$, where $n$ is large and $p>0$. In this case the Metallic Ratio of order $k$ will take the form $\rho_{k}=\frac{1+\sqrt{1+4 n^{2 p}}}{2 n^{2 p}}$.

Now considering the limit as $n \rightarrow \infty$ so that $k \rightarrow 0$

$$
\rho_{k}=\frac{1+\sqrt{1+4 n^{2 p}}}{2 n^{2 p}}=\frac{1}{2} \frac{1}{n^{2 p}}+\frac{1}{2} \sqrt{4+\frac{1}{n^{2 p}}} \rightarrow \frac{1}{2} \times 0+\frac{1}{2} \sqrt{4+0}=1 \text {. }
$$

Thus the Metallic Ratio approach 1 when $k$ is very small of the order $\frac{1}{n^{p}}$.

We observe from section 3 (Generalized Ratio), that the preciousness of ratios (like that of metals) decreases in increasing values of $k$. If $k=1$, we get Golden Ratio. So in order to get Rhodium and Platinum, the most prominent and precious metals than Gold, we can reduce the value of $k$ from 1 to further down say of the order $\frac{1}{n^{2}}, \frac{1}{n}$ respectively.

In particular if $k=\frac{1}{n^{2}}$ we get the Rhodium Ratios given by $\rho_{k}=\frac{1+\sqrt{1+4 n^{4}}}{2 n^{2}}, n=2,3,4, \ldots$

If $k=\frac{1}{n}$ we get the Platinum Ratios given by $\rho_{k}=\frac{1+\sqrt{1+4 n^{2}}}{2 n}, n=2,3,4, \ldots$

Further, from $\rho_{k}=\frac{k+\sqrt{k^{2}+4}}{2}$ we see that $\frac{\rho_{k+1}}{\rho_{k}} \rightarrow 1$ as $k \rightarrow \infty$. This means that for large values of $k$, the $k^{\text {th }}$ metallic ratio is almost identical to $(k+1)^{\text {th }}$ metallic ratio.

\section{Conclusions}

Introducing Golden Ratio, which is very familiar, I extended it to define other ratios such as Silver Ratio and Bronze Ratio. In this Paper, I attempted to generalize such ratios to define more generalized ratios which in turn can be used to determine new numbers called Platinum Ratio and Rhodium Ratio which were not dealt before. In this sense, this paper provides a new insight in understanding more general class of "Metallic Ratios". Moreover these new numbers will provide an ample opportunity for future research.

\section{Applications}

The Golden Ratio finds abundance application in all branches of Engineering and Technology. The number Golden Ratio has been used from Ancient time and it continues to get used in modern times. In particular, in constructing great Egyptian Pyramids and Parthenon Structure in Greece, this glorious Golden Ratio is used extensively. Similarly, the silver ratio finds application in Paper Sizes of all levels like A, B and C series. It is also quite interesting that one subtracted from Silver Ratio is the first irrational number to be discovered in the world.

Great Renaissance artists like Leonardo Da Vinci, Michelangelo, Luca Pacioli, Albrecht Durer used these metallic ratios quite extensively to produce memorable paintings. In particular, Golden and Silver ratios were chosen to decide their canvas sizes as well as to increase the aesthetic aspects of their paintings. Needless to say, these paintings have become the icon of human achievements in Arts.

Golden Ratio and Silver Ratios also appear at various places in nature. A whole book spanning about thousand pages can be written to brief about these applications themselves. Nature most often chooses spiral shapes to have efficient storage of particles and these spirals emanate from Golden Ratio. We often see those spirals in big galaxies and tiny DNA molecules. Many interesting applications and aspects of Golden Ratio and Metallic Ratios are available in [4], [6] and [13].

In this paper, by reducing the value of $k$ in the generalized metallic ratio, the resulting numbers resemble the preciousness and rarity of the corresponding metals. Thus the metallic ratios presented here mimic the exact life scenario, again proving the connection between numbers and elements of the universe, a primitive idea envisaged by Pythagoras some 2600 years ago.

\section{REFERENCES}

[1] Generalized Metallic Means, Juan B. Gil and Aaron Worley, Fibonacci Quarterly, Volume 57 (2019), Issue. 1, 45-50.

[2] SedaYamak, Akbiyik, Salim Yuce, On Metallic Ratio in $Z_{p}$, Special Issue: International Conference on Mathematics: An Istanbul meeting for mathematicians 2018, 15 November $2019,5535-5550$. 
[3] K. Hare, H. Prodinger, and J. Shallit,Three series for the generalized golden mean, Fibonacci Quart.52(2014), no. 4, 307-313.

[4] DannPassoja,Reflections on the Gold, Silver and Metallic Ratios, 2015.

[5] P. J. Cameron, Some sequences of integers, Discrete Math. 75(1989), 89-102.

[6] The Fabulous Fibonacci Numbers, Alfred Posamentier, Prometheus Publishers, First Edition, 2007

[7] Markowsky G. Misconceptions about the golden ratio. The College Mathematics Journal, 1992; 23:2-19.

[8] Fischler R., How to find the golden number without really trying, Fibonacci Quarterly, 1981; 19: 406-410.

[9] Newell AC, Pennybacker M. Fibonacci patterns: common or rare? Procedia IUTAM. 2013; 9(Supplement C):86-109.

[10] Krcadinac V., A new generalization of the golden ratio. Fibonacci Quarterly, 2006; 44(4):335-340.

[11] CoxeterHSM., Introduction to Geometry, 2nd Edition, Wiley; 1989.

[12] Stewart Ian, The Mathematics of Life, Basic Books; 2011.

[13] Mario Livio, The Golden Ratio: The story of Phi, the world's most astonishing number, First Edition, October 2002. 